\title{
0ITOMOMICS
}

Revista de los Estudios de Economía y Empresa

EDITORIAL

\section{Redes sociales, economía y empresa}

\section{Oriol Miralbell Izard}

Profesor de los Estudios de Economía y Empresa (UOC) y editor del dosier "Redes sociales, economía y empresa"

No hay duda de que una de las actividades que ha ido cambiando más en los últimos años en todo el mundo han sido las relaciones sociales, especialmente a través de las redes sociales online como Facebook, Twitter, Youtube, etc. La mayoría de usuarios que interactúan dentro de estas redes sociales lo hacen con diferentes propósitos, pero especialmente, para socializar y mantener relaciones con sus compañeros, familiares y amigos (Miralbell, 2014). Ahora bien, las oportunidades y los retos que las redes sociales online plantean abarcan de manera significativa la actividad económica global. Las tecnologías de la información y la comunicación (TIC) estimulan el dinamismo innovador (Lladós, Jiménez y Garay, 2008) y se han convertido en uno de los principales fundamentos del proceso de cambio radical que experimenta la economía y estructura social en la última década, porque devienen un recurso de uso masivo y de aplicación para todos los agentes económicos y sociales, y porque son la base del nuevo sustrato innovador que "transforma radicalmente la estructura de inputs básicos y costes relativos para la producción" (Torrent y Sellens, 2009).

En una economía globalizada y en el entorno de la sociedad red, en la que las dinámicas de la estructura social se generan a partir de redes activadas por el intercambio de información procesada digitalmente y por el uso de las tecnologías de la información y la comunicación (Castells, 2009), las empresas necesitan aprovechar la transferencia de conocimiento, tanto para su mejora competitiva como para la innovación de sus productos o servicios y de sus procesos productivos. En este ámbito, las redes de interacción tienden a crearse en torno a las estructuras organizadas y los agentes más decisivos e importantes para la economía global, mientras que los de menor relevancia, en función de su menor peso, irán ocupando puestos menos estratégicos y más periféricos, pudiendo, incluso quedar fuera de la red de la economía global.

La creación de valor es un proceso de producción organizado en torno a las TIC (lo mismo si se trata de la producción de bienes o de la realización de servicios) con unas tareas de toma de decisión de la innovación, de análisis, planificación, diseño y ejecución llevadas a cabo por diferentes perfiles profesionales que irían desde los mandos, pasando por los investigadores, diseñadores, integradores y operadores (Castells, 1997).

Los flujos de información para el conocimiento están condicionados en buena parte por la posición de los agentes en la red, y esto determinará también su capacidad de acceso y el control sobre esta información. Por ello, en la economía, factores como la capacidad tecnológica o el acceso a los mercados condicionan y dependen a la vez de la posición que tienen los agentes económicos en la red. Cuanto mejores sean los recursos tecnológicos mejor será la posición del agente, y a la vez, más interés tendrá en preservar su conocimiento entre los demás agentes con los que le interese compartirlo o haya colaborado en su generación. La competencia estimula la generación de nuevo conocimiento, que permite preservar la posición de preferencia de sus propietarios en la red. Esto no quita que aún quede camino por recorrer en la mayoría de empresas en lo que respecta al fomento entre sus trabajadores para que socialicen a través de las redes sociales, aunque los estudios existentes han demostrado que ello favorece que se comparta conocimiento, mejorando el rendimiento de la empresa y de sus profesionales (Miralbell, 2013). Todavía hay barreras que frenan que las empresas fomenten el uso de las redes sociales online tanto internamente como en las relaciones con otras empresas, entre las que se encuentra la 
dificultad para medir los beneficios de este uso y el desconocimiento de las oportunidades que ofrecen las redes sociales para las empresas (Jussila, Kärkkäinen, y Aramo-Immonen, 2014).

Dada la actualidad que tienen las redes sociales y con la intención de aportar más información a los usos y oportunidades que las redes sociales ofrecen a la economía, hemos recogido en el dosier de este segundo número de la revista Oikonomics cinco aportaciones de expertos que plantean diferentes aproximaciones a la relación de las redes sociales con la economía y la empresa.

En el primer artículo, Agustí Canals, profesor de los Estudios de Ciencias de la Información y de la Comunicación de la UOC y director del grupo de investigación KIMO, nos expone la influencia que tiene la estructura de las redes sociales en la eficiencia de la transmisión y la generación de conocimiento en las organizaciones. En el artículo se explica cómo, a través del uso de las redes sociales y de facilitar que estas organicen adoptando una estructura más adecuada a sus intereses, las organizaciones pueden gestionar mejor el conocimiento. En este sentido, Agustí Canals hace algunas reflexiones sobre las características de las redes sociales que más inciden en la consecución de los objetivos de las organizaciones.

En el segundo artículo, Inma Rodríguez-Ardura, profesora de los Estudios de Economía y Empresa de la UOC y directora del grupo de investigación Observatorio de la Nueva Economía (ONE), da respuesta a algunas preguntas que los especialistas de marketing se hacen a la hora de planificar una estrategia de marketing de medios sociales efectiva. Ella explica cómo dar forma a este tipo de estrategias de marketing digital, indicando cuáles son las diferentes fases y cómo evaluar los resultados.

En el tercer artículo de este dosier, Montserrat Peñarroya, consultora de los Estudios de Economía y Empresa de la UOC, cuenta diferentes usos que las empresas pueden hacer de las redes sociales online. Además de usarlas en el ámbito del marketing, las organizaciones pueden utilizarlas para la formación de clientes y trabajadores, para el apoyo a la formación, como entorno de relaciones entre los trabajadores o entre colectivos de dirección, para la búsqueda de nuevos directivos y para la gestión de los recursos humanos, entre otros.

Desde una perspectiva más global de la economía, Elisabeth Ruiz, profesora de los Estudios de Economía y Empresa de la UOC y miembro del grupo de investigación Observatorio de la Nueva Economía (ONE), plantea en el cuarto artículo los factores que intervienen en la comunicación global y sus efectos en los mercados financieros. A la vez, ilustra cómo uno de los factores más relevantes a la hora de la integración de los mercados financieros ha sido el avance tecnológico que ha hecho posible la conexión entre mercados, que entre otros ha contribuido a agilizar la negociación con servicios integrados de varios de aquellos mejorando el binomio riesgorendimiento para los inversores. Fruto de estos cambios en el sistema financiero y su dinámica de comunicación, ha habido una evolución de los productos financieros existentes y la aparición de nuevos. Elisabeth Ruiz nos habla también del aspecto negativo de la globalización y la elevada interconexión entre mercados, como es el efecto contagio de las crisis financieras.

Finalmente, la entrevista nos ofrece la visión de un experto como Enrique Dans, profesor de Sistemas de Información en IE Business School y autor de varios artículos y libros sobre la sociedad de la información, en los que habla de la evolución de las TIC y de las redes sociales, y su impacto en las organizaciones y en la economía global.

Para dar a conocer los mejores trabajos de los estudiantes de los Estudios de Economía y Empresa de la UOC, además del dosier, presentamos un artículo sobre un Trabajo Final de Grado en Administración y Dirección de Empresas, que ha sido seleccionado por su excelencia. María Francisca Manrique nos hace una síntesis del trabajo donde, a través del análisis integral de una empresa del sector del curtido de la piel, ha querido determinar los factores clave que afectan a la misión de la empresa.

En definitiva, confiamos en que esta segunda edición de la revista Oikonomics, en la que se ofrece al lector una aproximación de cómo las redes sociales inciden en la economía y cómo las empresas pueden aprovecharlas para mejorar su competitividad, sea de interés. 


\section{Bibliografía}

CASTELLS, M. (1997). «La Sociedad Red». La era de la información. Vol. 1. Madrid: Alianza Editorial.

CASTELLS, M. (2009). Communication power. Oxford University Press Oxford.

JUSSILA, J. J.; KÄRKKÄINEN, H.; ARAMO-IMMONEN, H. (2014). «Social media utilization in business-to-business relationships of technology industry firms". Computers in Human Behavior. N. ${ }^{\circ}$ 30, págs. 606-613.

LLADÓS, J.; JIMÉNEZ, A. I.; GARAY, L. (2008). «La innovació digitlal de l'empresa catalana: patrons, característiques i dterminants». En: L'Empresa Xarxa. Tecnologies de la Informació i la Comunicació. Productivitat i Competitivitat (J. Vilaseca i J. Torrent.). Barcelona: Ariel.

MIRALBELL, O. (2013). "Las TIC y la transferencia de conocimiento en el turismo». En: Nuevos horizontes en la gestión del turismo. Págs. 57-81. Escola Universitària de Turisme i Gestió Hotelera, UAB.

MIRALBELL, O. (2014). «Knowledge Exchange in Social Networking Sites». En: Perspectives on Social Media: A Yearbook. Págs. 11-18. Routledge.

TORRENT I SELLENS, J. (2009). «Coneixement, xarxes i activitat econòmica: una anàlisi dels efectes de xarxa en l'economia del coneixement». UOC Papers. N. ${ }^{\circ}$ 8, págs. 1-23.

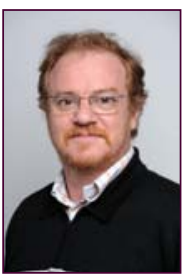

\section{Oriol Miralbell Izard}

Profesor de los Estudios de Economía y Empresa (UOC) y editor del dosier "Redes sociales, economía y empresa"

Doctor en Información y Sociedad del Conocimiento por la Universitat Oberta de Catalunya. Licenciado en Filología Alemana, máster en Gestión de la Información por la Universidad de Syracuse. Es profesor de los Estudios de Economía y Empresa de la UOC. Ha sido responsable de los servicios de información de la Dirección General de Turismo de la Generalitat de Cataluña. Es presidente del Capítulo español de la International Federation for IT and Travel \& Tourism (IFFIT), miembro del grupo de investigación GRATET (http://www.urv.cat/dgeo/gratet/) sobre Turismo y Geografía, miembro del Laboratorio del Nuevo Turismo (http: //turismo.blogs. uoc. edu / 3-recerca-id /). Centra su investigación en las TIC y el turismo, la gestión de los destinos y las redes sociales y el conocimiento.

Los textos publicados en esta revista están -si no se indica lo contrario- bajo una licencia Reconocimiento-Sin obras derivadas 3.0 España de Creative Commons. Puede copiarlos, distribuirlos y comunicarlos públicamente siempre que cite su autor y la revista y la institución que los publica (autoría, nombre de la revista, institución editora); no haga con ellos obras derivadas. La licencia completa se puede consultar en http://creativecommons.org/licenses/by-nd/3.0/es/deed.es.

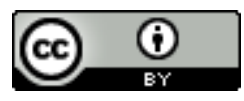

